Marquette University

e-Publications@Marquette

$9-12-2019$

\title{
Empirical Mappings of the Frequency Response of an Electron Ratchet to the Characteristics of the Polymer Transport Layer
}

\author{
Mohamad S. Kodaimati \\ Northwestern University \\ Ofer Kedem \\ Marquette University, ofer.kedeem@marquette.edu \\ George C. Schatz \\ Northwestern University \\ Emily A. Weiss \\ Northwestern University
}

Follow this and additional works at: https://epublications.marquette.edu/chem_fac

Part of the Chemistry Commons

\section{Recommended Citation}

Kodaimati, Mohamad S.; Kedem, Ofer; Schatz, George C.; and Weiss, Emily A., "Empirical Mappings of the Frequency Response of an Electron Ratchet to the Characteristics of the Polymer Transport Layer" (2019). Chemistry Faculty Research and Publications. 1007.

https://epublications.marquette.edu/chem_fac/1007 
Marquette University

e-Publications@Marquette

\section{Chemistry Faculty Research and Publications/College of Arts and Sciences}

This paper is NOT THE PUBLISHED VERSION; but the author's final, peer-reviewed manuscript. The published version may be accessed by following the link in the citation below.

Journal of Chemical Physics : C, Vol. 123, No. 36 (12 September 2019): 22050-22057. DOI. This article is (C) American Chemical Society Publications and permission has been granted for this version to appear in e-Publications@Marquette. American Chemical Society Publications does not grant permission for this article to be further copied/distributed or hosted elsewhere without the express permission from American Chemical Society Publications.

\section{Empirical Mappings of the Frequency Response of an Electron Ratchet to the Characteristics of the Polymer Transport Layer}

Mohamad S. Kodaimati

Center for Bio-Inspired Energy Science, Northwestern University, 303 E. Superior Street, 11th Floor, Chicago, Illinois

Department of Chemistry, Northwestern University, 2145 Sheridan Rd., Evanston, Illinois Ofer Kedem

Center for Bio-Inspired Energy Science, Northwestern University, 303 E. Superior Street, 11th Floor, Chicago, Illinois

Department of Chemistry, Northwestern University, 2145 Sheridan Rd., Evanston, Illinois

George C. Schatz 
Center for Bio-Inspired Energy Science, Northwestern University, 303 E. Superior Street, 11th Floor, Chicago, Illinois

Department of Chemistry, Northwestern University, 2145 Sheridan Rd., Evanston, Illinois

\section{Emily A. Weiss}

Center for Bio-Inspired Energy Science, Northwestern University, 303 E. Superior Street, 11th Floor, Chicago, Illinois

Department of Chemistry, Northwestern University, 2145 Sheridan Rd., Evanston, Illinois

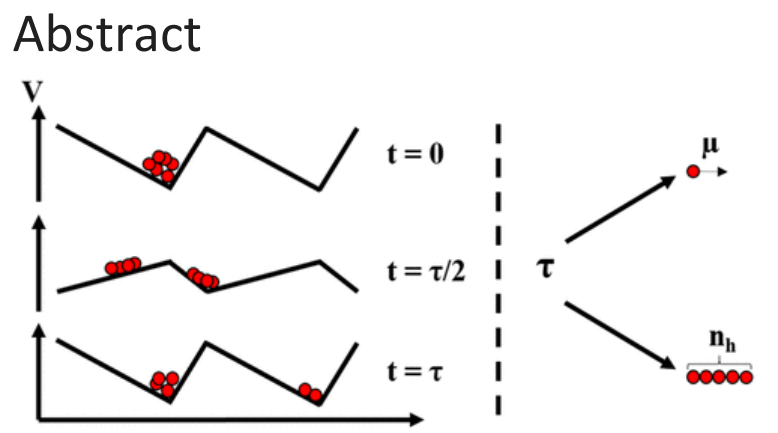

Flashing electron ratchets oscillate a periodic asymmetric potential to rectify nondirectional forces and thereby produce directional transport of electrons with zero source-drain bias. The relationship between the oscillation frequency of the potential and the ratchet (short-circuit) current reflects microscopic mechanisms of charge transport within the device. This paper describes experimental mappings of the "optimal frequency(ies)" of the ratchet $f_{\text {peak }}$-the oscillation frequencies that produce the largest ratchet current-to the carrier concentration, $n_{\mathrm{h}}$, and to the linear field effect transistor mobility, $\mu_{\mathrm{h}}$, for a poly(3-hexylthiophene-2,5-diyl) (P3HT) transport layer. Measurements on multiple devices, multiple P3HT films per device, and a range of annealing and photoexcitation conditions yield the empirical relationships $f_{\text {peak }} \propto n_{\mathrm{h}}$ and $f_{\text {peak }} \propto \mu_{\mathrm{h}}{ }^{2 / 3}$. Finiteelement simulations suggest the sublinear relationship between mobility and peak frequency arises due to a combination of damped and inertial motion of the holes. This work also provides evidence that the frequency response of ratchets is sensitive to multiple length scales of asymmetry encoded within the periodic electrical potential. These multiple asymmetries cause changes in the polarity of the ratchet current at points within the frequency response, a long-mysterious characteristic of particle ratchets called "current inversion", by encouraging transport in opposite directions in different frequency regimes.

\section{Introduction}

Particle ratchets are driven nonequilibrium systems that rectify nominally nondirectional (isotropic) forces to produce directional transport of particles, including electrons, by breaking both temporal symmetry and spatial inversion symmetry in the direction of transport. Molecular motors in biological systems - such as Na-K ATPase,(1) the myosin family,(2) and the kinesin family $(3,4)$-utilize a ratcheting mechanism; well-defined energetic landscapes defined by the structure and conformational states of the protein yield directional transport while hydrolyzing adenosine triphosphate (ATP). One class of synthetic ratchet, the flashing ratchet, utilizes a periodic array of asymmetric structural features, the "ratchet potential", which oscillates between two states of different symmetries to rectify the isotropic motion of a collection of particles (Figure 1).(5-8) The ratchet potential is typically electromagnetic and is used to drive the motion of various charged particles, including microspheres $(9,10)$ and electrons.(11-13) Flashing electron ratchets produce current without application of a source-drain bias and can be used in applications such as rectifiers, digital logic, cryptography, and potentially (if the energy input is light) solar energy conversion. What is needed to move toward these 
applications are experimental studies that map parameters of the electron ratchet to their electrical characteristics and performance and offer at least semiquantitative physical models for these mappings.

\section{Particle density}
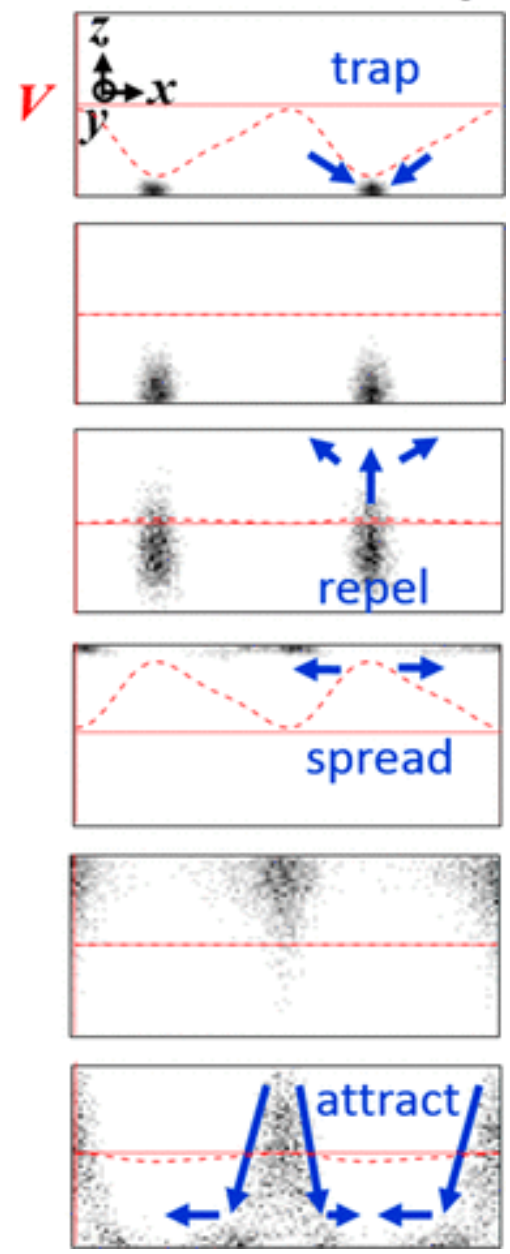

Figure 1. General mechanism of a 2D flashing particle ratchet: density plots displaying trajectories of likecharged nanoparticles in a medium of fixed dynamic viscosity $\left(1 \times 10^{-4} \mathrm{~Pa}\right.$ s) and dielectric constant (2.09) during application of a sinusoidal oscillating potential at the bottom boundary. Trajectories are calculated through finite element simulations. Darker areas indicate higher particle densities. The red dashed traces correspond to the potential applied, $V$. The $z$-axis indicates both the physical coordinate of the particles (spanning the thickness of the transport layer) and the electrostatic energy they experience, while the $x$-axis is the direction along which transport is recorded to determine particle current. The Supporting Information contains details of the simulation.

A major feature of any flashing particle ratchet, electron or otherwise, is a peaked dependence of the ratchet current or average particle velocity on the frequency of the oscillation of the ratchet potential. This peaked dependence is due to a change in the degree of coincidence between the time scale of oscillation and the inherent average time scale of motion of the traveling particles with changing oscillation frequency.(14-16) If the potential oscillation is too fast relative to particle motion, the particle experiences a time-averaged (spatially symmetric) potential, and there is no ratcheting. If the potential oscillation is too slow, the energy provided by the oscillation is dissipated as the particle relaxes to an equilibrium position at the bottom of a potential well, and there is no ratcheting. An examination of the frequency dependence of a flashing ratchet therefore offers insight into the time scale(s) of microscopic motion of the ratcheted particles, but so far there have been few 
reports experimentally relating the peak frequency of an electron ratchet and electrical properties of the transport layer.(17,18)

A second major feature of a ratchet is the dependence of ratchet current on the density and interaction potential of ratchet particles. Theoretical studies of repulsively interacting particles (e.g., hard sphere(19-23) and Coulombic repulsion(24)) have revealed a strong dependence of the ratchet current on particle density, identifying regimes of cooperative transport and destructive particle jamming, but there have been no experimental studies of the relationship of the frequency response and the carrier density for a particular type of transport layer.

A third characteristic of ratchets is that the polarity of the ratchet current does, under some conditions, switch with a change in oscillation frequency of the potential. These so-called "current inversions" have been mysterious signatures of both experimental and theoretical ratchets for the lifetime of the field.

Here, we use extensive experimental characterization of a flashing electron ratchet with a poly(3hexylthiophene-2,5-diyl) (P3HT) transport layer to propose mechanisms underlying all three of these ratchet characteristics. By varying the concentration and mobility of the majority carrier, holes, within P3HT through a combination of illumination and $\mathrm{CS}_{2}$ vapor annealing, we show that the "optimal" frequency of the ratchet (the frequency at which the highest short-circuit current is achieved) scales linearly with the carrier concentration and as the $2 / 3$ power of the linear field effect transistor mobility of the film. We also provide the first experimental evidence that the frequency response of the ratchet current depends on spatial asymmetries of multiple length scales within the ratchet potential, as opposed to only the spatial asymmetry of the fundamental repeating unit of the potential. The multiple asymmetries account for current inversions, since (i) asymmetries on different length scales in some cases encourage transport in opposite directions and (ii) asymmetries on longer length scales promote ratcheting at slower frequencies than asymmetries on shorter length scales.

\section{Experimental Methods}

\section{Device Architecture}

We fabricated ratchet devices with similar structures to those in our previous reports(14,15) (Figure $2 A, B)$; the fabrication procedure and device characterization are described in detail in the Supporting Information. Within these devices, we used focused ion beam (FIB) assisted Pt deposition to form (unless otherwise noted) eight asymmetrically shaped finger electrodes (FEs) on $\mathrm{Si} / \mathrm{SiO}_{2}$ substrates and encapsulated them with a dielectric layer composed of plasma-enhanced chemical vapor-deposited (PECVD) $\mathrm{SiO}_{2}$ and spin-on glass. On top of the dielectric layer, we deposited $\mathrm{Ag}$ source and drain electrodes using photolithography and spin-coated P3HT transport layers ( $\sim 70 \mathrm{~nm}$ thick), which cover the $12 \mu \mathrm{m}$ channel between the source and drain electrodes. By applying a potential to the FEs (versus the source electrode), we generate an electric potential within the transport layer, $U(r)$, which is determined by both the shape of the FEs (Figure $2 \mathrm{C}$ ) and the dielectric response of the device. The time-dependent ratchet potential, $V(r, t)$, experienced by the carriers within the transport channel is a product of $U(r)$ and $F(t)$, the time-dependent sinusoidal waveform that depends on the amplitude of the potential, $A$, and the flashing frequency, $f$ (eq 1 ).

$$
\begin{aligned}
& V(r, t)=U(r) F(t)(1 \mathrm{a}) \\
& F(t)=A \sin (2 \pi t f)(1 \mathrm{~b})
\end{aligned}
$$


In this work, the amplitude of the potential, $A$, is $10 \mathrm{~V}$. We measure the ratchet current (current at zero sourcedrain bias) at a range of flashing frequencies, $f$, for four devices denoted D1 through D4 with very similar FE shapes, as verified by atomic force microscopy (AFM) scans (Figure $2 \mathrm{C}$ and Figure $\mathrm{S} 1$ ).

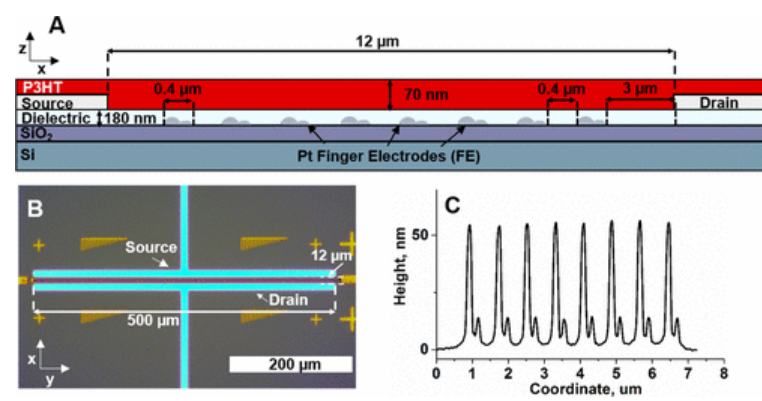

Figure 2. (A) Schematic diagram of a ratchet device where eight asymmetric Pt finger electrodes (FEs) encapsulated by a dielectric layer are used to generate an electric field within the transport layer (dimensions not to scale). (B) Optical microscopy image of the active area of one device, D1, prior to deposition of P3HT. The ratcheting transport channel is $12 \mu \mathrm{m}$ thick (between source and drain) and $50 \mathrm{~nm}$ high. (C) AFM line scan of the Pt FEs of device D1.

\section{Tuning the Electrical Properties of P3HT}

We employed two methods for tuning the conductivity of our spin-coated P3HT films after the deposition: (i) illumination of the $\mathrm{P} 3 \mathrm{HT}$ film with $532 \mathrm{~nm}$ light and (ii) annealing in $\mathrm{CS}_{2}$ vapor. We measured the dc conductivity $(\sigma)$ of the P3HT films by recording source-drain current $\left(I_{S D}\right)$ as a function of source-drain bias $\left(V_{S D}\right)$. We estimated the FET mobility $\left(\mu_{h}\right)$ of the films from linear-regime field-effect transistor (FET) mobility sweeps, specifically by applying a dc potential to the FEs, which serve as a gate (see the Supporting Information for details). The carrier concentration, $n_{\mathrm{h}}$, of our films is defined by eq 2

$\sigma=n_{\mathrm{h}} e \mu_{\mathrm{h}}(2)$

where $e$ is the fundamental charge of the electron. We assume that holes (rather than electrons) are responsible for the electrical response of the $\mathrm{P} 3 \mathrm{HT}$ films as $\mathrm{P} 3 \mathrm{HT}$ is known to be a p-type semiconductor,(25) and the mobility of electrons within our devices is more than a factor of 10 lower than that of the holes (see the Supporting Information). Illumination of the $\mathrm{P} 3 \mathrm{HT}$ film and $\mathrm{CS}_{2}$ vapor annealing each modifies both the mobility and carrier concentrations of $\mathrm{P} 3 \mathrm{HT}$ (Figures S3 and S4); thus, to assess the separate contributions of carrier concentration and hole mobility to the ratchet response, we need data sets where one of these parameters is held constant while the other one varies. We produce such data sets by varying the intensity of illumination and duration of $\mathrm{CS}_{2}$ annealing for a set of $\mathrm{P} 3 \mathrm{HT}$ films on each of four devices and then compare the electrical characteristics of ratchets with either constant $n_{\mathrm{h}}$ (within $\pm 25 \%$ ) or constant $\mu_{\mathrm{h}}$ (within $\pm 25 \%$ ). This strategy is possible because we can dissolve the spin-coated $\mathrm{P} 3 \mathrm{HT}$ films in $\mathrm{CHCl}_{3}$ and redeposit on the same device (see the Supporting Information). Through a combination of absorbance, photoluminescence, and Raman spectroscopy measurements, we determined that $\mathrm{CS}_{2}$ vapor annealing decreases the mobility of the P3HT films by disordering the packing structure of the spin-coated films (Figure S5). We assume, in our analysis, that the hole mobility of P3HT is spatially isotropic (see the Supporting Information for details).

\section{Results and Discussion}

\section{Effect of Carrier Mobility and Concentration on the Frequency Response of the Ratchet}

The $y$-axis of Figure $3 \mathrm{~A}$ is the net charge transported per oscillation of the ratchet, $q_{\text {osc }}$; this quantity scales the ratchet current by the frequency $(f)$ of the applied sinusoidal potential and is therefore a measure of ratchet 
efficiency. A plot of $q_{\text {osc }}$ vs $f$ is the frequency response of the ratchet. Figure 3A shows the frequency responses for a series of transport layers on a single device D1 (analogous plots for other devices are in the Supporting Information). For all of these films, the carrier concentration is $2.6 \times 10^{-3} \mathrm{C} \mathrm{cm}^{-3}( \pm 25 \%)$, and the hole mobility ranges from $7.7 \times 10^{-5}$ to $1.7 \times 10^{-3} \mathrm{~cm}^{2} \mathrm{~V}^{-1} \mathrm{~s}^{-1}$. There is no discernible trend in the magnitude of $q_{\mathrm{osc}}$ with $\mu_{\mathrm{h}}$ as a function of frequency.
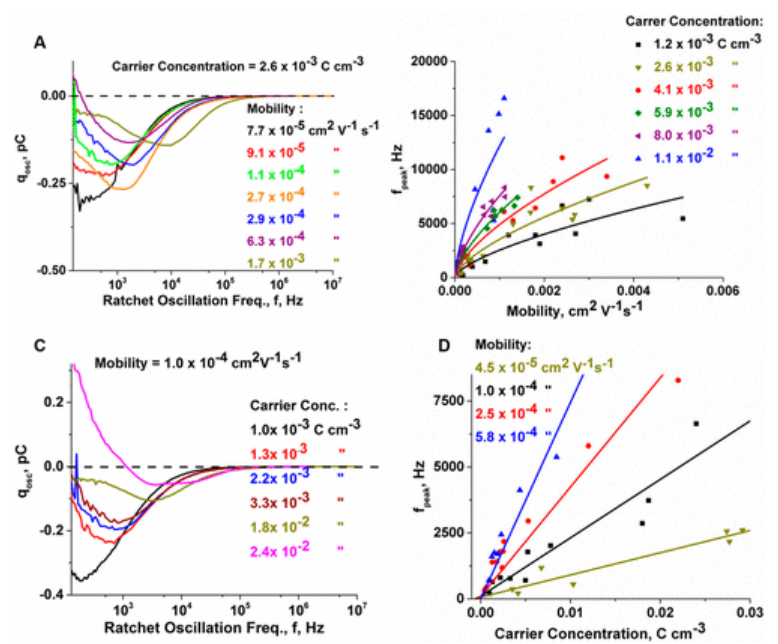

Figure 3. (A) Plots of source-drain charge as a function of ratcheting frequency for device D1 driven by $10 \mathrm{~V}$ sine waves with a fixed carrier concentration of $2.6 \times 10^{-3} \pm 6.5 \times 10^{-4} \mathrm{C} \mathrm{cm}^{-3}$, with differing mobilities. (B) Plot of peak ratcheting frequency vs linear FET mobility for devices D1-D4 with varying carrier concentrations. Solid traces are power law fits described in the main text. (C) Plots of source-drain charge as a function of ratcheting frequency for device D1 driven by $10 \mathrm{~V}$ sine waves, with a fixed linear FET mobility of $1.0 \times 10^{-4} \mathrm{~cm}^{2} \mathrm{~V}^{-1} \mathrm{~s}^{-1}$ with differing carrier concentrations. The trend of decreased overall ratchet current with increasing carrier concentration is likely due to electron-hole recombination, as explained in the Supporting Information. (D) Plot of peak ratcheting frequency vs carrier concentration for devices D1-D4 with mobilities of $4.5 \times 10^{-5}$ (yellow), 1 $\times 10^{-4}$ (black), $2.5 \times 10^{-4}$ (red), and $5.8 \times 10^{-4} \mathrm{~cm}^{2} \mathrm{~V}^{-1} \mathrm{~s}^{-1}$ (blue). Data for a mobility of $5.8 \times 10^{-4} \mathrm{~cm}^{2} \mathrm{~V}^{-1} \mathrm{~s}^{-1}$ are only plotted if the frequency response had a single discernible peak, as opposed to two peaks.

The most notable feature of these results is a maximum magnitude of $q_{\mathrm{f}}$ at a frequency that varies with mobility. We denote the oscillation frequency at which the absolute value of $q_{\text {osc }}$ is maximized as $f_{\text {peak. }}$. As the carrier mobility increases, $f_{\text {peak }}$ shifts to higher values, as shown for a single carrier concentration in Figure $3 \mathrm{~A}$ and summarized at a series of carrier concentrations $\left(n_{\mathrm{h}}\right)$ in Figure 3B for four devices, D1-D4. In our case, the sign of $q_{\text {osc }}$ is negative; however, we previously demonstrated that the direction of ratcheting is dependent upon the FE shape.(26) The relationship between $f_{\text {peak }}$ and $\mu_{\mathrm{h}}$ is sublinear in this range of $n_{\mathrm{h}}$. When we scale the traces in Figure $3 \mathrm{~B}$ by $n_{\mathrm{h}}$ and plot versus $\mu_{\mathrm{h}}$, the traces all overlay (Figure S8); this result indicates that $f_{\text {peak }}$ is linearly proportional to $n_{\mathrm{h}}$. Furthermore, plots of $f_{\text {peak }} / n_{\mathrm{h}}$ versus $\mu_{\mathrm{h}}$ are nonlinear. These two observations are summarized in eq 3

$f_{\text {peak }} \approx C n_{\mathrm{h}} f\left(\mu_{\mathrm{h}}\right)$

where $f\left(\mu_{h}\right)$ is a sublinear function of mobility. Through a global fit of the data in Figure 3B to a power law function of the form $f_{\text {peak }}=C_{i} n_{\mathrm{h},} \mu_{\mathrm{h}}{ }^{x}$, where $x$ is shared among all traces, $n_{\mathrm{h}, i}$ is the fixed carrier concentration for trace $i$, and $C_{i}$ is a free parameter for trace $i$, we empirically determine $x$ to be $0.66 \pm 0.06$ (eq 4).

$f_{\text {peak }} \approx C n_{\mathrm{h}} \mu_{\mathrm{h}}^{2 / 3}(4)$ 
The proportionality constant $C$ translates the mobility and carrier concentration into an average time scale of motion of the carriers and is primarily dictated by the drift velocity of the carriers during the ratcheting mechanism. The drift velocity, in turn, depends on the electric field experienced by the carriers; $C$ is therefore different for different FE shapes and dielectric layers (Figure S6).

If we instead measure a series of $\mathrm{P} 3 \mathrm{HT}$ ratchets with the same value of $\mu_{\mathrm{h}}( \pm 25 \%)$, but a range of values of $n_{\mathrm{h}}$ (Figure 3C), we examine the frequency response of the ratchet as a function of carrier concentration and independently verify eq 4 . At lower mobilities (here, $\leq 2.5 \times 10^{-4} \mathrm{~m}^{2} \mathrm{~V}^{-1} \mathrm{~s}^{-1}$ ), $f_{\text {peak }}$ indeed shifts linearly to higher values with increasing carrier concentration as defined by eq 4 (Figure 3D). Furthermore, with this independent data set, we observe a $2 / 3$ power dependence of $f_{\text {peak }}$ on carrier mobility, also consistent with eq 4 (Figure S8).

To understand the empirical relationship between $f_{\text {peak }}$ and $\mu_{\mathrm{h}}$ in eq $4,\left(f_{\text {peak }} \propto \mu_{\mathrm{h}}{ }^{2 / 3}\right)$ we refer to a previously published 1D analytical model for Brownian (overdamped) particles subject to an oscillating spatially asymmetric potential;(27) we have found this model useful in describing certain behaviors of our experimental flashing ratchets.(15) The 1D model predicts a linear relationship between the $f_{\text {peak }}$ and $\mu_{\mathrm{h}}$ (Figure S10). If we expand the 1D model to 2D by allowing diffusion of particles in the spatially homogeneous $y$-dimension, we also observe a linear relationship between $f_{\text {peak }}$ and $\mu_{\mathrm{h}}$ (see the Supporting Information). We therefore hypothesized that the observed sublinear dependence of $f_{\text {peak }}$ on $\mu_{\mathrm{h}}$ arises from either (i) motion of carriers in the $z$-dimension (thickness) of our transport layer in response to oscillation of the potential between positive and negative or (ii) inertial effects as the carriers are not in a strictly overdamped transport regime. To distinguish between these mechanisms, we examine 2-dimensional $(x, z)$ simulations of charged particles in solution experiencing an oscillating spatially asymmetric potential, where we vary the diffusivity/mobility of the particles by adjusting the dynamic viscosity of the solvent. For low simulated mobilities $\left(\sim 7 \times 10^{-5}\right.$ to $\left.7 \times 10^{-4} \mathrm{~cm}^{2} \vee \mathrm{s}^{-1}\right)$, we observe a linear relationship between the peak ratcheting frequency and mobility (Figure S11A,B), indicating that our experimentally observed sublinear relationship does not arise from the motion of the carriers in the $z$-direction. At higher mobilities $\left(\sim 7 \times 10^{-4}\right.$ to $\left.1.2 \times 10^{-3} \mathrm{~cm}^{2} \mathrm{~V}^{-1} \mathrm{~s}^{-1}\right)$, we observe a broad frequency response, where the peak frequency does not depend upon the mobility (Figure S11C). In this mobility regime, the particles no longer experience overdamped transport and-due to inertial effects-are less spatially confined by the ratcheting potential (Figure S12). Assuming the ratcheting current, $I$, scales as $f^{-1}$ in the high-frequency limit(27) and $f$ in the low-frequency limit,(28) we can use a simple ansatz to characterize the frequency dependence of the ratcheting current (eq 5)

$I(f, \tau) \approx \frac{f \tau^{-1}}{f^{2}+f \tau^{-1}+\tau^{-2}} \varphi(5)$

where $\tau$ corresponds to the time scale of transport of a particle across one spatial period, $L$, of the ratcheting potential and $\phi$ is a proportionality constant with units of current. Qualitatively, eq 5 expresses the current in terms of $\phi$ times the ratio of productive ratchet driven motion (the $f^{-1}$ term) to total motion, with the $f^{2}$ term corresponding to unproductive motion in which the frequency term applies twice (first pushing one direction and then the reverse), and the $\tau^{-2}$ term refers to motion not driven by the ratchet that could include both ballistic and diffusive components. At steady state the current $l$ is proportional to the charge $q$, so to arrive at an expression for $f_{\text {peak, }}$, the extrema of $q_{\text {osc, }}$ we divide eq 5 by $f$ and set the derivative (eq 6 )

$$
\frac{\partial}{\partial \mathrm{f}} \mathrm{q}_{\mathrm{osc}}(f, \tau) \approx \frac{\tau^{-2}+2 f \tau^{-1}}{\left(f^{2}+\frac{f}{\tau}+\tau^{-2}\right)^{2}} \varphi(6)
$$

equal to zero, yielding $f_{\text {peak }} \approx \frac{\tau^{-1}}{2}$. In the case of inertial/ballistic transport, $\tau \propto \frac{\mathrm{L}}{\sqrt{\mathrm{U}}}$, where $U$ is the ratcheting potential experienced by the particle, whereas $\tau \propto \frac{L^{2}}{D}$ in the overdamped limit, where $D$ is the 
diffusivity/mobility of the particle. Therefore, we would expect no dependence of $f_{\text {peak }}$ upon the particle mobility and a linear dependence of $f_{\text {peak }}$ upon the particle mobility in the case of underdamped/inertial and overdamped transport, respectively. The sublinear behavior in our experimental ratchets could be attributed to hole transport within the P3HT having mixed overdamped and inertial transport character.

Previous simulations of charged particle ratchets have shown that at low carrier concentrations carrier-carrier repulsion effects lead to a stronger potential experienced by the particles, increasing the peak frequency; at high carrier concentrations, the carrier-carrier repulsion begins to damp particle transport-so-called "jamming"giving an overall peaked dependence of $f_{\text {peak }}$ on $n_{\mathrm{h}}$. $(21,22)$ Our observed approximately linear relationship between $f_{\text {peak }}$ and $n_{\mathrm{h}}$ at mobilities less than $1.1 \times 10^{-3} \mathrm{~cm}^{2} \mathrm{~V}^{-1} \mathrm{~s}^{-1}$ (eq 4 ) is reasonable if we are not achieving sufficient carrier densities to result in the jamming effects seen in simulations. Our hole densities are limited primarily by recombination of holes with less mobile electrons. The existence of electron-hole recombination in these films is supported by both (i) a sublinear relationship between carrier concentration and illumination intensity and (ii) a decrease in measured hole mobility upon illumination (see Figure S3B). Future experimental work can validate this hypothesis by comparing the effects of carriers introduced by chemical or electrochemical doping versus photogenerated carriers.

At higher mobilities $\left(>8.2 \times 10^{-4} \mathrm{~cm}^{2} \mathrm{~V}^{-1} \mathrm{~s}^{-1}\right)$, the frequency response of the ratchet is more complex: we still observe a shift of the response to higher frequencies with increasing $n_{\mathrm{h}}$, but the response also broadens and eventually splits into multiple discernible peaks (Figure 4A,B). We suspect these effects are due to the fact that when measuring the FET mobility, we preferentially measure holes with the highest mobility (a known problem in characterizing organic semiconductors(29)), but populations of holes with lower mobilities exist in the film. As we increase $n_{\mathrm{h}}$, the holes sample a broad distribution of morphologies (amorphous and crystalline regions) within the heterogeneous P3HT film, and therefore have a set of distributions of time scales of motion. Each distribution gives rise to a peak in the frequency response of the ratchet. 


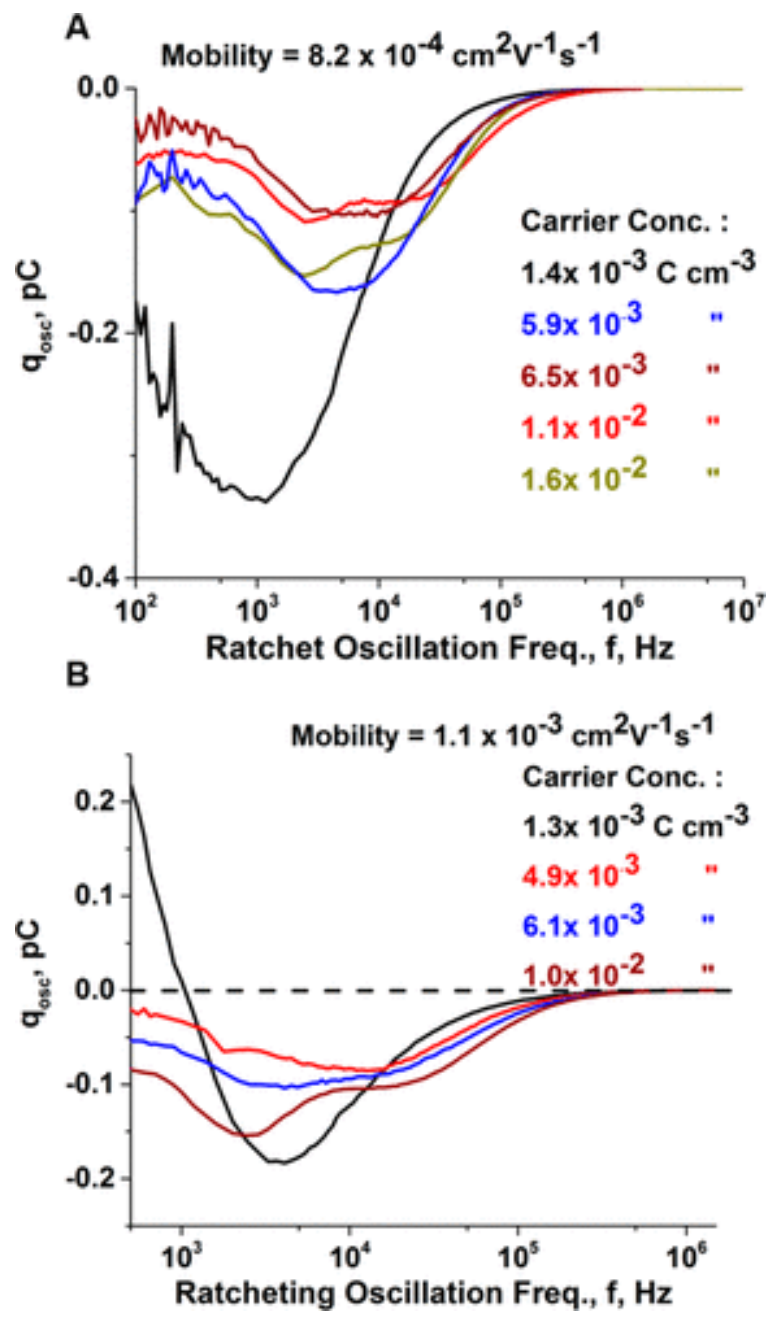

Figure 4. (A) Plots of source-drain charge as a function of ratcheting frequency for device D1 driven by $10 \mathrm{~V}$ sine waves, with a fixed linear FET mobility of $8.2 \times 10^{-4} \mathrm{~cm}^{2} \mathrm{~V}^{-1} \mathrm{~s}^{-1}$ with differing carrier concentrations. (B) Plots of source-drain charge as a function of ratcheting frequency for device D1 driven by $10 \mathrm{~V}$ sine waves, with a fixed linear FET mobility of $1.1 \times 10^{-3} \mathrm{~cm}^{2} \mathrm{~V}^{-1} \mathrm{~s}^{-1}$ with differing carrier concentrations. All mobility values are within $25 \%$ of the specified value.

\section{Sensitivity of Ratchets to Multiple Length Scales of Spatial Asymmetry: One Origin of Current Inversions}

In addition to the shape and peak position of the frequency response, the other major electrical characteristic of a flashing electron ratchet is the current inversion, where the directionality of particle transport is inverted $(30,31)$ on going from one frequency region to another. Clear examples of such inversions are in Figure $3 \mathrm{C}$ (pink trace) and Figure 4B (black trace). We view the observed ratchet current as the small net current produced from the competition between the current in the source-to-drain direction and the current in the drain-to-source direction. While the asymmetry in the shape of each FE is the intentional, engineered source of asymmetry in the system, this asymmetry is very small once the field propagates into the transport layer (see Figure S15). Other asymmetries in the potential surface therefore may also contribute to ratchet current, and these asymmetries may drive the current in the opposite direction of that dictated by the shape of individual FEs. One such "incidental" asymmetry is caused by an underlying curvature of the ratchet potential surface in the $x$-direction (Figure 5A). Finite-element simulations of the electric potential generated by the FEs at the $\mathrm{P} 3 \mathrm{HT} / \mathrm{SiO}_{2}$ interface predict this curvature and show that it is caused by the difference in the electrical 
potential around FEs in the center of the array from FEs near the ends of the array. This curvature creates an additional unit of asymmetry associated with two or more adjacent FEs (termed a multi-FE mode). Since this unit of asymmetry has a longer period than the individual FE mode, holes moving in response to this asymmetry are resonant with a slower oscillation frequency than holes moving in response to the asymmetry of a single FE. If the multi-FE mode and the single-FE ratchet in opposite directions, the result is a current inversion.

Figure 5
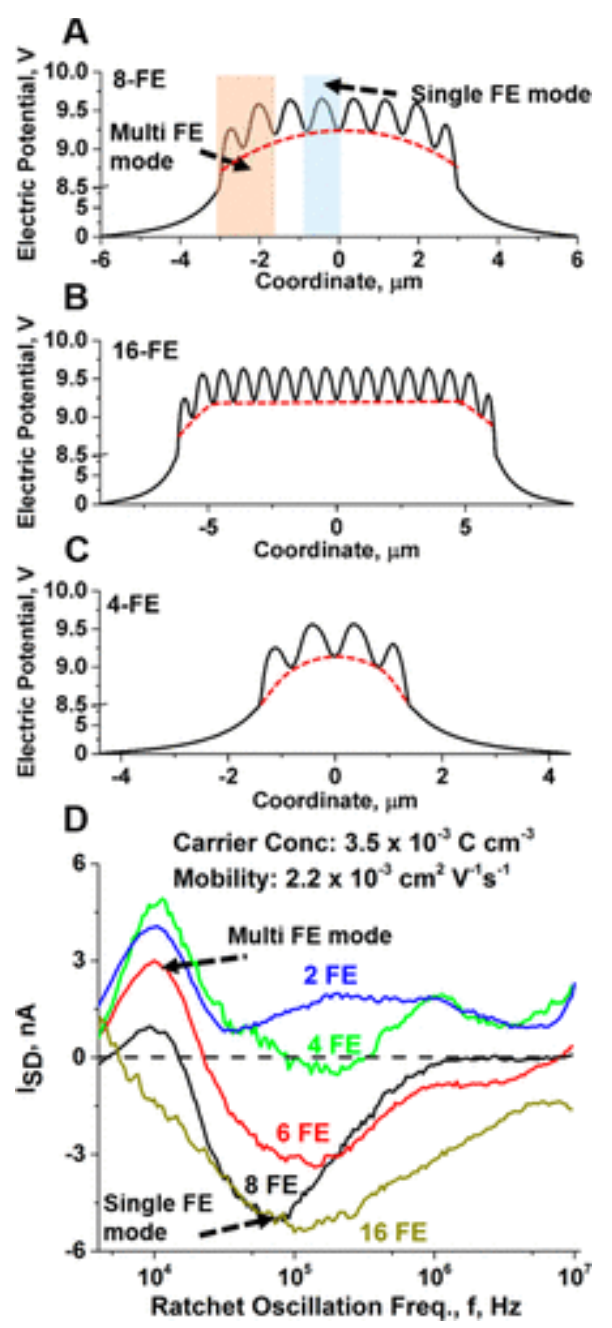

Figure 5. Plots of simulated electric fields versus $x$ coordinate at the $\mathrm{P} 3 \mathrm{HT} / \mathrm{SiO}_{2}$ interface for 8-FE (A), 16-FE (B), and 4-FE (C) devices. Red dashed lines highlight the longer length scale curvature of the potential surface. (D) Plots of source-drain current at zero applied source-drain bias as a function of ratcheting frequency for devices with 16 (yellow), 8 (black), 6 (red), 4 (green), and 2 (blue) FEs and driven by $10 \mathrm{~V}$ sine waves with fixed carrier concentration $\left(3.5 \times 10^{-3} \mathrm{C} \mathrm{cm}^{-3}\right)$ and mobility $\left(2.2 \times 10^{-3} \mathrm{~cm}^{2} \mathrm{~V}^{-1} \mathrm{~s}^{-1}\right)$.

To confirm this hypothesis, we fabricated devices with 2, 4, 6, 8, or 16 FEs, where the devices all have very similar FE shapes, $400 \mathrm{~nm}$ separation between FEs, and $3 \mu \mathrm{m}$ separating the source (drain) electrode from the first (last) FE (see the Supporting Information for details). Increasing the number of FEs decreases the curvature of the underlying potential (e.g., compare Figures 5A-5C) and should decrease the contribution of the multi-FE mode to the overall frequency response of the ratchet.

Figure 5D shows the frequency responses of this set of devices, all of which we engineered to have a common $n_{\mathrm{h}}$ and $\mu_{\mathrm{h}}( \pm 25 \%)$; here, we plot source-drain current, $l_{\mathrm{sd}}$, rather than charge as we are primarily 
interested in the different regimes of transport (rather than their efficiencies). All of the responses exhibit an artifactual peak at $\sim 3 \mathrm{kHz}$ that we attribute to capacitance of the FEs and source/drain electrodes; we subtracted this feature from the data sets (see the Supporting Information for details). When we decrease the number of FEs from 16 to 8 to 6 to 4 , the magnitude of the negative higher frequency peak $\left(\sim 10^{5} \mathrm{~Hz}\right)$ (which we tentatively attribute to the single-FE mode) decreases and appears to shift to higher frequencies, and the magnitude of the positive lower frequency peak $\left(\sim 10^{4} \mathrm{~Hz}\right)$ (which we tentatively attribute to a multi-FE mode) increases. The frequency at which the current inversion occurs also increases with a decreasing number of FEs. One can interpret these trends as a competition between ratcheting in the negative current direction due to asymmetry of the single FEs and ratcheting in the positive current direction due to asymmetries on a longer length scale. As the curvature of the ratchet potential increases (on going from 16 to $4 \mathrm{FEs}$ ), the contribution of the lower frequency response increasingly dominates the contribution of the higher frequency response. In the "2 FE" device, the single FE mode completely disappears, and we observe two broad positive peaks.

We observe these trends across different mobilities and different carrier concentrations (see Figure S16).

\section{Conclusions}

In summary, we examined the relationship between the ratcheting frequency and three microscopic properties in flashing electron ratchets with a $\mathrm{P} 3 \mathrm{HT}$ transport layer: (i) carrier mobility, (ii) carrier concentration, and (iii) spatial asymmetry across multiple length scales. By varying the concentration and mobility of the holes within the film through a combination of illumination and $\mathrm{CS}_{2}$ annealing, we observe that the peak ratcheting frequency, the frequency of oscillation that provides the biggest ratchet current, scales linearly with the carrier concentration and as the $2 / 3$ power of the linear FET mobility. We provide evidence that increasing carrier concentration via photoexcitation increases the peak ratcheting frequency through repulsive carrier-carrier interactions. We also provide evidence that the sublinear relationship between the mobility and peak frequency arises from inertial effects, where carrier transport is no longer in an overdamped regime. Finally, we demonstrate that spatial asymmetries across multiple length scales (multi-FE modes) contribute to the ratcheting response and, in some cases, induce a current reversal in this response.

In our experimental system, we introduce free carriers through illumination-where the photogenerated holes are more mobile and primarily responsible for the ratcheting response. By using a transport layer with more ambipolar transport behavior (such as amorphous $\mathrm{Si}(32)$ or $\mathrm{WSe}_{2}(33)$ ), we would potentially increase the peak ratcheting efficiency by (i) finding an oscillation frequency that resonates with both electron and hole motion and (ii) decreasing electron-hole recombination events by spatially separating the two carriers. Additionally, by electrically doping the ratchet device through a top contact, we could avoid electron-hole recombination entirely and possibly introduce enough carriers to induce theoretically predicted jamming effects. While, in our system, the carrier mobility is isotropic (in-plane vs out-of-plane are not differentiable), portions of the racheting process consist of primarily horizontal or vertical transport (Figure 1). We could gain insight into the time scales of these substeps in the ratcheting process through the use of anisotropic transport layers, such as transition metal dichalcogenides (TMDCs)(34) or highly ordered semiconductor polymers.(35)

While the multi-FE modes arise in our system due to our devices' poorly approximating an infinite periodic array of FEs, one could imagine intentionally encoding multiple length scales of asymmetry within a ratchet device. In our devices, we observed a current inversion arising from the multi-FE mode; however, we could potentially encode ratchets with tunable frequency responses across a wide frequency range. When constructively interfering with the single-FE mode, the multi-FE modes generate a broadband frequency response. When destructively interfering with the single-FE mode, they give rise to a narrowed frequency response. By properly mapping the functional dependence of the frequency response versus the carrier mobility, carrier concentration, and spatial asymmetry within the field (including multiple length scales of asymmetry), we can 
potentially fabricate ratchet information devices with tunable ratcheting efficiencies for a wide frequency range ( $100 \mathrm{~Hz}$ to $10 \mathrm{MHz}$ ) using our current device structure.

\section{Supporting Information}

The Supporting Information is available free of charge on the ACS Publications website at DOI: $10.1021 /$ acs.jpcc.9b06503.

Device fabrication and characterization, methodology for electrical measurements, optical characterization of P3HT (absorption, photoluminescence, and Raman spectra), analytical simulations, and finite-element simulations (PDF)

The authors declare no competing financial interest.

\section{Terms \& Conditions}

Electronic Supporting Information files are available without a subscription to ACS Web Editions. The American Chemical Society holds a copyright ownership interest in any copyrightable Supporting Information. Files available from the ACS website may be downloaded for personal use only. Users are not otherwise permitted to reproduce, republish, redistribute, or sell any Supporting Information from the ACS website, either in whole or in part, in either machine-readable form or any other form without permission from the American Chemical Society. For permission to reproduce, republish and redistribute this material, requesters must process their own requests via the RightsLink permission system. Information about how to use the RightsLink permission system can be found at http://pubs.acs.org/page/copyright/permissions.html.

\section{Acknowledgments}

This work was supported as part of the Center for Bio-Inspired Energy Science (CBES), an Energy Frontier Research Center funded by the U.S. Department of Energy (DOE), Office of Science, Basic Energy Sciences (BES), under Award \# DE-SC0000989. This work made use of the Keck-II and EPIC facilities of the NUANCE Center at Northwestern University, which has received support from the Soft and Hybrid Nanotechnology Experimental (SHyNE) Resource (NSF ECCS-1542205); the MRSEC program (NSF DMR-1720139); the International Institute for Nanotechnology (IIN); the Keck Foundation; and the State of Illinois, through the IIN. This work utilized Northwestern University Micro/Nano Fabrication Facility (NUFAB), which is partially supported by SHyNE, the Materials Research Science and Engineering Center, the State of Illinois, and Northwestern University. The authors thank Daniel Kwasnieski for useful discussions.

\section{References}

1 Tsong, T. Y. Na,K-Atpase as a Brownian Motor: Electric Field-Induced Conformational Fluctuation Leads to Uphill Pumping of Cation in the Absence of Atp. J. Biol. Phys. 2002, 28, 309-325, DOI: 10.1023/A:1019991918315

2 Shiroguchi, K.; Kinosita, K. Myosin V Walks by Lever Action and Brownian Motion. Science 2007, 316, 1208-1212, DOI: 10.1126/science.1140468

3 Leduc, C.; Padberg-Gehle, K.; Varga, V.; Helbing, D.; Diez, S.; Howard, J. Molecular Crowding Creates Traffic Jams of Kinesin Motors on Microtubules. Proc. Natl. Acad. Sci. U. S. A. 2012, 109, 6100-6105, DOI: 10.1073/pnas.1107281109

4 Shao, Q.; Gao, Y. Q. On the Hand-over-Hand Mechanism of Kinesin. Proc. Natl. Acad. Sci. U. S. A. 2006, 103, 8072-8077, DOI: 10.1073/pnas.0602828103

5 Lau, B.; Kedem, O.; Schwabacher, J.; Kwasnieski, D.; Weiss, E. A. An Introduction to Ratchets in Chemistry and Biology. Mater. Horiz. 2017, 4, 310-318, DOI: 10.1039/C7MH00062F 
6 Astumian, R. D.; Bier, M. Fluctuation Driven Ratchets: Molecular Motors. Phys. Rev. Lett. 1994, 72, 1766, DOI: 10.1103/PhysRevLett.72.1766

7 Sanchez-Palencia, L. Directed Transport of Brownian Particles in a Double Symmetric Potential. Phys. Rev. E 2004, 70, 011102, DOI: 10.1103/PhysRevE.70.011102

8 Sjölund, P.; Petra, S. J. H.; Dion, C. M.; Jonsell, S.; Nylén, M.; Sanchez-Palencia, L.; Kastberg, A. Demonstration of a Controllable Three-Dimensional Brownian Motor in Symmetric Potentials. Phys. Rev.

Lett. 2006, 96, 190602, DOI: 10.1103/PhysRevLett.96.190602

9 Rousselet, J.; Salome, L.; Ajdari, A.; Prostt, J. Directional Motion of Brownian Particles Induced by a Periodic Asymmetric Potential. Nature 1994, 370, 446-447, DOI: 10.1038/370446a0

10 Tierno, P.; Fischer, T. M. Excluded Volume Causes Integer and Fractional Plateaus in Colloidal Ratchet Currents. Phys. Rev. Lett. 2014, 112, 1, DOI: 10.1103/PhysRevLett.112.048302

11 Müller, T.; Würtz, A.; Lorke, A.; Reuter, D.; Wieck, A. D. Wave-Form Sampling Using a Driven Electron Ratchet in a Two-Dimensional Electron System. Appl. Phys. Lett. 2005, 87, 042104, DOI: 10.1063/1.2001740

12 Mikhnenko, O. V.; Collins, S. D.; Nguyen, T. Q. Rectifying Electrical Noise with an lonic-Organic Ratchet. Adv. Mater. 2015, 27, 2007- 2012, DOI: 10.1002/adma.201404450

13 Drexler, C.Magnetic Quantum Ratchet Effect in Graphene. Nat. Nanotechnol. 2013, 8, 104- 107, DOI: 10.1038/nnano.2012.231

14 Kedem, O.; Lau, B.; Ratner, M. A.; Weiss, E. A. Light-Responsive Organic Flashing Electron Ratchet. Proc. Natl. Acad. Sci. U. S. A. 2017, 114, 8698-8703, DOI: 10.1073/pnas.1705973114

15 Kedem, O.; Lau, B.; Weiss, E. A. How to Drive a Flashing Electron Ratchet to Maximize Current. Nano Lett. 2017, 17, 5848- 5854, DOI: 10.1021/acs.nanolett.7b03118

16 Bier, M.; Astumian, R. D. Biasing Brownian Motion in Different Directions in a 3-State Fluctuating Potential and an Application for the Separation of Small Particles. Phys. Rev. Lett. 1996, 76, 4277-4280, DOI: 10.1103/PhysRevLett.76.4277

17 Roeling, E. M.; Germs, W. C.; Smalbrugge, B.; Geluk, E. J.; de Vries, T.; Janssen, R. A. J.; Kemerink, M. Scaling of Characteristic Frequencies of Organic Electronic Ratchets. Phys. Rev. B: Condens. Matter Mater. Phys. 2012, 85, 045430, DOI: 10.1103/PhysRevB.85.045430

18 Roeling, E. M.; Germs, W. C.; Smalbrugge, B.; Geluk, E. J.; de Vries, T.; Janssen, R. A. J.; Kemerink, M. Organic Electronic Ratchets Doing Work. Nat. Mater. 2011, 10, 51- 55, DOI: 10.1038/nmat2922

19 Aghababaie, Y.; Menon, G.; Plischke, M. Universal Properties of Interacting Brownian Motors. Phys. Rev. E: Stat. Phys., Plasmas, Fluids, Relat. Interdiscip. Top. 1999, 59, 2578, DOI: 10.1103/PhysRevE.59.2578

20 Chacko, J.; Tripathy, G. Interacting Particles in Disordered Flashing Ratchets. Indian J. Phys. 2015, 89, 981-988, DOI: 10.1007/s12648-015-0660-5

21 Chakraborty, D.; Chaudhuri, D. Stochastic Ratcheting of Two-Dimensional Colloids: Directed Current and Dynamical Transitions. Phys. Rev. E 2015, 91, 050301, DOI: 10.1103/PhysRevE.91.050301

22 Derenyi, I.; Ajdari, A. Collective Transport of Particles in a "Flashing" Periodic Potential. Phys. Rev. E: Stat. Phys., Plasmas, Fluids, Relat. Interdiscip. Top. 1996, 54, R5, DOI: 10.1103/PhysRevE.54.R5

23 Einax, M.; Solomon, G. C.; Dieterich, W.; Nitzan, A. Unidirectional Hopping Transport of Interacting Particles on a Finite Chain. J. Chem. Phys. 2010, 133, 054102, DOI: 10.1063/1.3463000

24 Kedem, O.; Weiss, E. A. Cooperative Transport in a Multi-Particle, Multi-Dimensional Flashing Ratchet. J. Phys. Chem. C 2019, 123, 6913-6921, DOI: 10.1021/acs.jpcc.9b00344

25 Cunningham, P. D.; Hayden, L. M. Carrier Dynamics Resulting from above and Below Gap Excitation of P3ht and P3ht/Pcbm Investigated by Optical-Pump Terahertz-Probe Spectroscopy. J. Phys. Chem.

C 2008, 112, 7928-7935, DOI: 10.1021/jp711827g

26 Lau, B.; Kedem, O.; Ratner, M. A.; Weiss, E. A. Identification of Two Mechanisms for Current Production in a Biharmonic Flashing Ratchet. Phys. Rev. E: Stat. Phys., Plasmas, Fluids, Relat. Interdiscip.

Top. 2016, 93, 062128, DOI: 10.1103/PhysRevE.93.062128 
27 Rozenbaum, V. M. High-Temperature Brownian Motors: Deterministic and Stochastic Fluctuations of a Periodic Potential. JETP Lett. 2008, 88, 342- 346, DOI: 10.1134/S0021364008170128

28 Rozenbaum, V. M.; Makhnovskii, Y. A.; Shapochkina, I. V.; Sheu, S.-Y.; Yang, D.-Y.; Lin, S. H. Inertial Effects in Adiabatically Driven Flashing Ratchets. Phys. Rev. E 2014, 89, 052131, DOI: 10.1103/PhysRevE.89.052131

29 Choi, H. H.; Cho, K.; Frisbie, C. D.; Sirringhaus, H.; Podzorov, V. Critical Assessment of Charge Mobility Extraction in Fets. Nat. Mater. 2018, 17, 2- 7, DOI: 10.1038/nmat5035

30 Linke, H.; Humphrey, T. E.; Löfgren, A.; Sushkov, A. O.; Newbury, R.; Taylor, R. P.; Omling, P. Experimental Tunneling Ratchets. Science 1999, 286, 2314- 2317, DOI: 10.1126/science.286.5448.2314

31 Reichhardt, C. J. O.; Reichhardt, C. Ratchet Effects in Active Matter Systems. Annu. Rev. Condens. Matter Phys. 2017, 8, 51- 75, DOI: 10.1146/annurev-conmatphys-031016-025522

32 van Berkel, C.; Powell, M. J. Resolution of Amorphous Silicon Thin-Film Transistor Instability Mechanisms Using Ambipolar Transistors. Appl. Phys. Lett. 1987, 51, 1094- 1096, DOI: 10.1063/1.98751

33 Wang, Z.; Li, Q.; Chen, Y.; Cui, B.; Li, Y.; Besenbacher, F.; Dong, M. The Ambipolar Transport Behavior of WSe ${ }_{2}$ Transistors and Its Analogue Circuits. NPG Asia Mater. 2018, 10, 703- 712, DOI: 10.1038/s41427018-0062-1

34 Ghorbani-Asl, M.; Enyashin, A. N.; Kuc, A.; Seifert, G.; Heine, T. Defect-Induced Conductivity Anisotropy in $\mathrm{MoS}_{2}$ Monolayers. Phys. Rev. B: Condens. Matter Mater. Phys. 2013, 88, 245440, DOI: 10.1103/PhysRevB.88.245440

35 O’Connor, B. T.; Reid, O. G.; Zhang, X.; Kline, R. J.; Richter, L. J.; Gundlach, D. J.; DeLongchamp, D. M.; Toney, M. F.; Kopidakis, N.; Rumbles, G. Morphological Origin of Charge Transport Anisotropy in Aligned Polythiophene Thin Films. Adv. Funct. Mater. 2014, 24, 3422-3431, DOI: 10.1002/adfm.201303351 